\title{
23rd International Conference on Dialysis: Advances in Chronic Kidney Disease 2021 (April 20-23, 2021, USA)
}

\author{
Peter Kotanko ${ }^{a, b}$ Laura Rosales ${ }^{a}$ Claudio Ronco ${ }^{c-e}$ \\ ${ }^{a}$ Renal Research Institute, New York, NY, USA; ${ }^{b}$ Icahn School of Medicine at Mount Sinai, New York, NY, USA; \\ 'Department of Medicine, University of Padova, Padova, Italy; ${ }^{\mathrm{d}}$ International Renal Research Institute of \\ Vicenza, Vicenza, Italy; ${ }^{\text {e }}$ epartment of Nephrology, San Bortolo Hospital, Vicenza, Italy
}

\section{Advances in Chronic Kidney Disease 2021}

This year's special issue of the Renal Research Institute's 23rd International Conference on Dialysis, Advances in Chronic Kidney Disease (CKD) is dedicated to all healthcare professionals that are at the forefront of combatting the COVID-19 pandemic and impacted by its global burden. It is exemplary how healthcare workers worldwide were able to overcome challenges with extraordinary resilience. Our CME-accredited 23rd International Conference Program is available throughout the year on various social media platforms (https://www. facebook.com/RenalResInstitute; https://twitter.com/ RR_Institute; https://www.linkedin.com/company/therenal-research-institute/?viewAsMember=true; https:// www.youtube.com/channel/UCFy_E0plIIINd0LOMP0iCWA).

For the first time the Renal Research Institute's Conference was held in a virtual format. The conference accomplished an in-depth and diverse scientific program for clinicians, nephrologists, researchers, nurses, nephrology pediatricians, and fellow professionals in training, delivered by skillful scientists, physicians, and experts in the field. Thanks to the opportunities afforded by

karger@karger.com

(c) 2021 S. Karger AG, Basel

www.karger.com/bpu

Karger a virtual environment, we decided to release ahead of the conference some important and timely lectures related to the pandemic. It provided an update on topics from the latest on COVID-19 research, clinical nephrology, and basic science, to the most recent technological innovations and novel treatments in acute kidney injury (AKI), $\mathrm{CKD}$, hemodialysis, peritoneal dialysis, kidney, and transplantation.

In this issue of Blood Purification Topf and Williams [1] provide a compelling review on COVID-19 and social media. The latter of which could be "equally efficient at spreading information as misinformation". People who use it as their primary source of information are more vulnerable to misleading information. The authors bring into context the role of the "Public Physician" who connects and engages directly with other physicians and a diverse public, allowing them to debate topics, and potentially identify false information as it spreads. The authors interviewed several prominent professionals who during the pandemic needed to redirect their efforts to combat coronavirus "infodemic".

In their research article Han et al. [2] analyzed the impact of lockdown and social distancing measures on physical activity levels of hemodialysis (HD) patients. HD 
patients are already at an increased risk of reduced physical activity and further limitation could be detrimental. As Han et al. report, their urban population of patients had a $20 \%$ decrease in physical activity following lockdown measures compared to the pre-COVID-19 baseline. Interestingly, patients diagnosed with COVID-19 infection had increased activity levels before and during the incubation period compared to their COVID-19 negative counterparts.

Patients with COVID-19 may have an increased risk of kidney disease. Kooman and van der Sande [3] wrote a clear and detailed review of the clinical aspects of $\mathrm{HD}$ patients with COVID-19 and AKI in patients without prior kidney disease. The authors expertly placed a clinical challenge into focus, and we learned the latest insights into COVID-19 and kidney pathophysiology, incidence, clinical course, treatment, and outcomes.

Critically ill patients who require renal replacement therapy have high mortality. Pattharanitima et al. [4] report their results on predicting renal replacement therapy-free survival in critically ill patients with AKI. The authors used publicly available data from the Medical Information Mart for Intensive Care III, compiled over 12 years from intensive care unit admissions in a single center and applied 7 models of machine learning algorithms. Their study showed that artificial intelligence (AI) is a valuable tool for predicting outcomes and that it can surpass traditional models.

Han et al. [5] investigated hepatitis B vaccination response in HD patients retrospectively. As evidence has shown altered immune function in this patient population, finding ways to optimize vaccination was what motivated the authors to explore factors associated with seroconversion, such as indicators of sleep duration. Their analysis in a vast population of patients from a U.S. national dialysis provider found that early dialysis start time was associated with higher odds of seroconversion in univariate analysis. The authors hypothesize that a better sleep after vaccination may be responsible for this observation, similar to results observed in healthy subjects.

The interesting and original investigation by Krackov et al. [6] describes a method to perform an automatic and swift classification of vascular access (VA) aneurysms using $\mathrm{AI}$ on pictures taken by a smartphone. Current VA monitoring techniques to assess aneurysms may fail to recognize AV aneurysms. Therefore, the authors set out to develop an effective AI-based system to classify VA images.

Sleep apnea (SA) is prevalent in CKD and end-stage kidney disease (ESKD) patients. Zoccali et al. [7] summa- rize elegantly in this narrative review the current knowledge regarding its pathophysiology, risk factors and cardiovascular risk factors, and treatments, including the evolution of SA after kidney transplantation. The authors pose an important question: whether SA predicts adverse clinical outcomes in renal transplant patients.

The review article by Mclaren et al. [8] focuses on measuring and monitoring patient-reported outcomes such as pain and depression and how they relate to quality of life. The authors agree that patient-centered care could address specific issues more effectively and efficiently by providing the patients with a voice in their own care. Due to the COVID-19 pandemic, the use of telehealth and electronic patient-reported outcomes in the CKD and ESKD setting have increased dramatically.

The review article by Wood et al. [9] provides novel educational strategies to motivate dialysis patients to learn about living donor kidney transplantation and help lessen the burden on dialysis providers which usually have insufficient resources in this field. The authors describe the benefit of Digital Storytelling for health education which can be done affordably, using low-cost technology that most patients are already familiarized with.

The narrative review by Maierean and Oliver [10] on health outcomes and cost considerations of assisted peritoneal dialysis $(\mathrm{aPD})$ show results on the underutilization of aPD from different countries and regions from a variety of PD programs despite its cost-effectiveness. The authors focus on evaluating aPD programs and in-center HD with their corresponding barriers and opportunities.

The paper by Ikizler and Cuppari [11] on the 2020 updated KDOQI clinical practice guidelines for nutrition provides an excellent and comprehensive description of evidence-based recommendations intended to optimize CKD patient healthcare. The updated guidelines have been expanded to include adults with CKD stages $1-5$, on maintenance $\mathrm{HD}$ and $\mathrm{PD}$, and individuals with kidney transplant.

The mini review by Dupuis et al. [12] on plant-based diet elaborates on its potential benefits for HD patients to overcome known complications of the gastrointestinal tract such as constipation, improving phosphate balance, metabolic acidosis, and hyperkalemia.

An original investigation by Stavas et al. [13] evaluates the feasibility and safety of using renal autologous cell therapy injection in adults with CKD and corrected congenital anomalies of the kidney and urinary tract (CAKUT), where delaying a decline in kidney function is crucial. If effective, the novel therapy may have the potential of delaying kidney function decline in CAKUT patients. 
Advancing healthcare transition to adulthood in adolescents with CKD and ESKD has been a topic of interest of Diaz-Gonzalez de Ferris et al. [14] for many years. Their review provides a detailed account on the latest diverse approaches to accomplish optimal patient outcomes. We learn about prevalence and etiology of CKD and ESKD in adolescents and young adults which also manifests unique comorbid conditions affecting each organ system and ultimately influencing quality of life. Healthcare transition is challenging for caregivers and providers and demands an interdisciplinary approach starting in early adolescence and continuing towards adulthood.

Moura-Neto's [15] engaging article provides a multilevel strategy to face the issue of declining interest in training for a nephrology career, which has precipitated a shortage of nephrologists worldwide. Though the decision of choosing a specialty may be made early, other aspects such as practical work and research experience have been identified as positive motivators in the nephrology field.

The Renal Research Institute's steady dedication to advancing and strengthening therapeutic options for CKD and ESKD patients continues providing the finest medical care based on research and technological innovations. A commitment to improving patient outcomes and quality of life has made the Renal Research Institute the leader in the field. It has active national and international research programs with strong academic alliances with Columbia University in New York, NY; Weill Cornell Medicine, New York, NY; Icahn School of Medicine at Mount Sinai, New York, NY; St. Raphael Hospital/Yale, New Haven, CT; University of Massachusetts, Lowell, MA; University of North Carolina, Chapel Hill, NC; University of California Santa Barbara, CA; Pontifical Catholic University of Paraná, Curitiba, Brazil; Instituto de Cardiologia Ignacio Chavez, Mexico City, Mexico; the University of Guadalajara, Mexico; the University of
Maastricht, The Netherlands; Catharina Hospital Eindhoven, The Netherlands; University Medical Center Groningen, The Netherlands; Taipei Medical University Hospital, Taiwan; Karlsruhe Institute of Technology, Germany; University of Konstanz, Germany; Medizinische Universität Innsbruck, Austria; Joanneum Research Health, Graz, Austria and the University of Graz, Austria, among others. We hope that you will enjoy the wide range of papers published in the special issue of Blood Purification dedicated to the 23rd International Conference on Dialysis, Advances in Kidney Disease that took place virtually from April 20 to 23, 2021.

The Editors,

Peter Kotanko, Laura Rosales, and Claudio Ronco

\section{Acknowledgement}

We would like to thank Maggie Han for her expedited revision.

\section{Conflict of Interest Statement}

P.K. and L.R. are employees of the Renal Research Institute, a wholly owned subsidiary of Fresenius Medical Care North America. P.K. holds stock in Fresenius Medical Care. P.K. receives author honorarium from HS Talks. C.R. has no conflict of interest to declare.

\section{Funding Sources}

This preface and published articles were fully funded by the Renal Research Institute.

\section{Author Contributions}

The authors were responsible for the conception, preparation, drafting, and revision of the editorial.

\section{References}

1 Topf JM, Williams PN. COVID-19, Social Media, and the Role of the Public Physician. Blood Purif. 2021. DOI: 10.1159/000512707.

2 Han M, Preciado P, Thwin O, Tao X, TapiaSilva LM, Fuentes LR, et al. Effect of Statewide Lockdown in Response to COVID-19 Pandemic on Physical Activity Levels of Hemodialysis Patients. Blood Purif. 2021. DOI: $10.1159 / 000514935$.
3 Kooman JP, van der Sande FM. COVID-19 in ESRD and Acute Kidney Injury. Blood Purif. 2020. DOI: $10.1159 / 000513214$.

4 Pattharanitima P, Vaid A, Jaladanki SK, Paranjpe I, O’Hagan R, Chauhan K, et al. Comparison of Approaches for Prediction of Renal Replacement Therapy-Free Survival in Patients with Acute Kidney Injury. Blood Purif. DOI: 10.1159/000513700.
5 Han M, Ye X, Rao S, Williams S, Thijssen S, Hymes J, et al. Hepatitis B Vaccination Response in Hemodialysis Patients: The Impact of Dialysis Shift. Blood Purif. DOI: 10.1159/ 000513154

6 Krackov W, Sor M, Razdan R, Zheng H, Kotanko P. Artificial Intelligence Methods for Rapid Vascular Access Aneurysm Classification in Remote or In-Person Settings. Blood Purif. 2021. DOI: 10.1159/000515642. 
7 Zoccali C, Roumeliotis S, Mallamaci F. Sleep Apnea as a Cardiorenal Risk Factor in CKD and Renal Transplant Patients. Blood Purif. 2021. DOI: $10.1159 / 000513424$.

8 Mclaren S, Jhamb M, Unruh M. Using Patient-Reported Measures to Improve Outcomes in Kidney Disease. Blood Purif. 2021. DOI: 10.1159/000515640.

9 Wood EH, Waterman AD, Pines R. Storytelling to Inspire Dialysis Patients to Learn about Living Donor Kidney Transplant. Blood Purif. 2021. DOI: 10.1159/000512651.

10 Maierean SM, Oliver MJ. Health Outcomes and Cost Considerations of Assisted Perito- neal Dialysis: A Narrative Review. Blood Purif. 2021. DOI: 10.1159/000512839.

11 Ikizler TA, Cuppari L. The 2020 Updated KDOQI Clinical Practice Guidelines for $\mathrm{Nu}$ trition in Chronic Kidney Disease. Blood Purif. DOI: 10.1159/000513698.

12 Dupuis L, Brown-Tortorici A, Kalantar-Zadeh K, Joshi S. A Mini Review of Plant-Based Diets in Hemodialysis. Blood Purif. 2021. DOI: 10.1159/000516249.

13 Stavas J, Diaz-Gonzalez de Ferris M, Johns A, Jain D, Bertram T. Protocol and Baseline Data on Renal Autologous Cell Therapy Injection in Adults with Chronic Kidney Disease Sec- ondary to Congenital Anomalies of the Kidney and Urinary Tract. Blood Purif. 2021. DOI: $10.1159 / 000512586$.

14 Diaz-Gonzalez de Ferris ME, Díaz-González de Martínez ML, Díaz-González de Velázquez AM, Díaz-González Borja A, Díaz-González Borja A, Filler G, et al. An Interdisciplinary Approach to Optimize the Care of Transitioning Adolescents and Young Adults with CKD. Blood Purif. 2021. DOI: 10.1159/000513520.

15 Moura-Neto JA. "To Be, or Not to Be" a Nephrologist: Students' Dilemma and a Strategy for the Field. Blood Purif. 2021. DOI: $10.1159 / 000513155$. 